\title{
Finite-Difference Time-Domain Analysis of a Complete Transverse Electromagnetic Cell Loaded with Liquid Biological Media in Culture Dishes
}

\author{
Milica Popović,* Student Member, IEEE, Susan C. Hagness, Member, IEEE, and Allen Taflove, Fellow, IEEE
}

\begin{abstract}
Transverse electromagnetic (TEM) cells can be used for exposing biological culture specimens to electromagnetic fields and observing possible anomalous effects. The uniformity of field exposure is critical to quantifying the biological response versus the electromagnetic dose. Standing waves and other electromagnetic field nonuniformities can cause nonuniform exposure. This paper reports the results of high-resolution three-dimensional finite-difference time-domain (FDTD) simulations of a complete TEM cell designed for operation at $837 \mathrm{MHz}$. Several different cases were studied in which the number of culture dishes, the depth of the culture liquid, and the orientation of the culture dishes were varied. Further, the effect of the culture-dish glass bottom thickness and the meniscus of the liquid medium were examined. The FDTD results show that there is a significant nonuniform field and specific absorption rate (SAR) distribution within the culture medium for each case examined. Hence, biological dose-response experiments using the TEM cell should account for the possibility of strong localized SAR peaking in the culture media to provide useful data in setting exposure standards for wireless communications.
\end{abstract}

Index Terms - Absorbing media, biological cells, biological effects of electromagnetic radiation, biological system modeling, dosimetry, electromagnetic propagation in absorbing media, FDTD methods, waveguides.

\section{INTRODUCTION}

$\mathbf{N}$ OVEL wireless technologies have been very widely adopted for routine communications usage in our society. These technologies generally place the user in close proximity to radiated UHF and microwave fields having source powers of hundreds of milliwatts. Since the daily exposure of hundreds of thousands of people to electromagnetic fields of this intensity is without precedent, much concern and interest has been expressed in the possibility that there could be associated health risks.

Manuscript received June 6, 1997; revised February 10, 1998. This work was supported in part by Wireless Technology Research L.L.C. and Cray Research, Inc. The work of S. C. Hagness was supported in part by an NSF Graduate Fellowship. Asterisk indicates corresponding author.

*M. Popović is with the Department of Electrical and Computer Engineering, McCormick School of Engineering, Northwestern University, 2145 Sheridan Road, Evanston, IL 60208 USA (e-mail: poppy@nwu.edu).

S. C. Hagness is with the Department of Electrical and Computer Engineering, University of Wisconsin-Madison, Madison, WI 53706 USA.

A. Taflove is with the Department of Electrical and Computer Engineering, McCormick School of Engineering, Northwestern University, Evanston, IL 60208 USA.

Publisher Item Identifier S 0018-9294(98)05330-0.
One way to study possible health risks of human exposure to electromagnetic fields is to set up controlled experiments wherein living cell cultures are placed in a well-defined field environment and any cell pathology is carefully examined. In principle, this would permit an electromagnetic "dose"-biological response relation to be established. The controlled field environment should be easily replicable and relevant to actual human exposure conditions.

At radio frequencies, a well-understood field environment is that of a transverse electromagnetic (TEM) wave, that is, a uniform plane wave. The TEM wave is the simplest radiating electromagnetic wave, representing the limiting form of the energy radiated by an antenna in its far field. This wave can be generated in a closed, shielded structure in the laboratory called a TEM cell [1] shown in Fig. 1. The TEM cell is simply described as a section of rigid, air-insulated coaxial line wherein the inner conductor (the septum) is a long, flat plate and the outer conductor is a rectangular tube. RF energy flows longitudinally along the line. If the frequency of the RF source is bounded to cut off non-TEM waveguide modes, the TEM cell generates reasonably uniform electric $(E)$ and magnetic $(H)$ fields in any transverse cross section of the cell at points away from the edges of the septum. Flared transition sections from the relatively large rectangular cross section of the TEM cell to the adjoining narrow diameter, circular cross sections of flexible coaxial cable are provided to smooth the flow of energy from the RF source into the cell and to the matched load. Biological tissue cultures are placed within the TEM cell and exposed to the propagating fields. The culture dishes can either rest upon the septum if the TEM cell is positioned horizontally, or can be oriented perpendicular to the septum if the TEM cell is positioned vertically.

A key problem with this exposure arrangement is that unavoidable field nonuniformities within the TEM cell limit the precision of knowledge of the electromagnetic dose of each tissue culture specimen. These nonuniformities are due to: 1) longitudinal standing waves within the TEM cell; 2) field enhancements near the surface and edges of the septum; 3) wave reflections within individual tissue-culture dishes; and 4) wave reflections between adjacent tissue-culture dishes. In addition, electromagnetic field nonuniformities also lead directly to nonuniform temperatures within the culture dishes because of RF power absorption by the culture media. Separating the biological effects of tissue temperature from those 


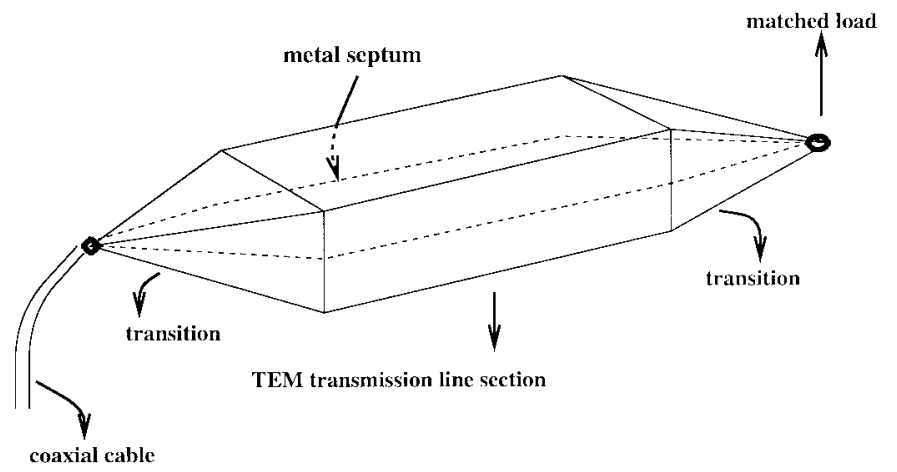

(a)

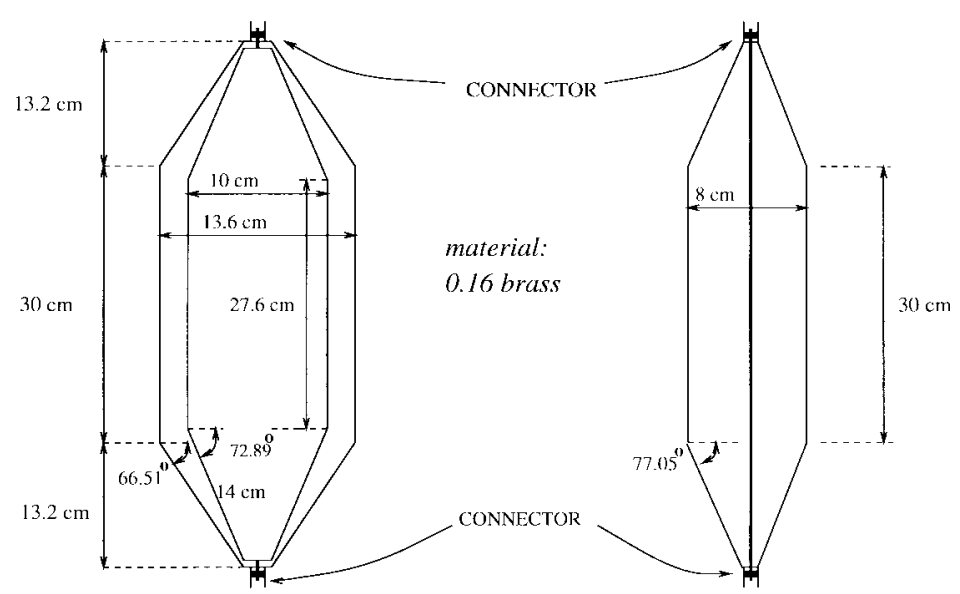

(b)

Fig. 1. (a) Perspective view of the TEM cell. (b) Geometry of the TEM cell. Left: plan view showing the septum; right: side cross section view.

of tissue field exposure is clearly complicated by exposure nonuniformity.

Since the publication of [1], little detailed modeling of the electromagnetic properties of TEM cells has appeared in the literature. The primary relevant publications [2], [3] reported finite-difference time-domain (FDTD) computational electromagnetics simulations [4]-[6] of only the rectangular part of the TEM cell, omitting the RF source, the input tapered transition section, the output tapered transition section, and the matched load. Excitation was provided in the model by assuming a TEM modal structure or static field distribution. The structure under test was taken to be a homogeneous cube with permittivity and conductivity equal to that of muscle tissue. Additional work on an engineering design approach of the TEM cell using the finite-element method was reported in [7].

The work reported in this paper explores the use of highresolution FDTD simulations to quantify electromagnetic field exposure nonuniformities within tissue-culture media dishes located in TEM cells. Electric field distributions obtained by the FDTD models permit subsequent mapping of the specific absorption rate (SAR) heating potential within the culture dishes. We make no assumptions regarding a modal structure of the field distribution, and utilize a full three-dimensional (3-D) FDTD model that includes the RF source, the input and output tapered transition sections, each culture dish and its contents, and the matched load. Modeling details of each culture dish include the shape and thickness of the polystyrene walls, the thickness of the borosilicate glass bottom, and the shape of the curved liquid surface due to the meniscus effect. Several baseline cases are investigated. First, a single dish is assumed to be filled with cell culture liquid and resting horizontally on the septum. The impact of the finite thickness of the culture-dish glass bottom is considered. Second, a horizontal-septum two-dish model is considered, with the cell culture filling only a portion of each dish. As a special case, the effect of including the liquid's meniscus upon the SAR distribution is examined. Third, the two culture dishes are assumed to be oriented perpendicular to a vertical septum. Here, the wavevector of the impinging field is perpendicular to the liquid surface. These FDTD simulations indicate that the electromagnetic field exposure nonuniformity is potentially significant for both the cases of the culture dishes resting on the septum and perpendicular to the septum.

\section{MaXwell's EQuations AND THE FDTD Method}

The propagation of electromagnetic waves within the TEM cell and the consequent electromagnetic field interactions with the biological tissue cultures are governed by Maxwell's equations. Due to the complicated nature of the geometry of the TEM cell and the culture dishes, the full 3-D set of E- and $\mathrm{H}$-field vector components must be involved. In this research, the FDTD numerical modeling method [4]-[6] is 

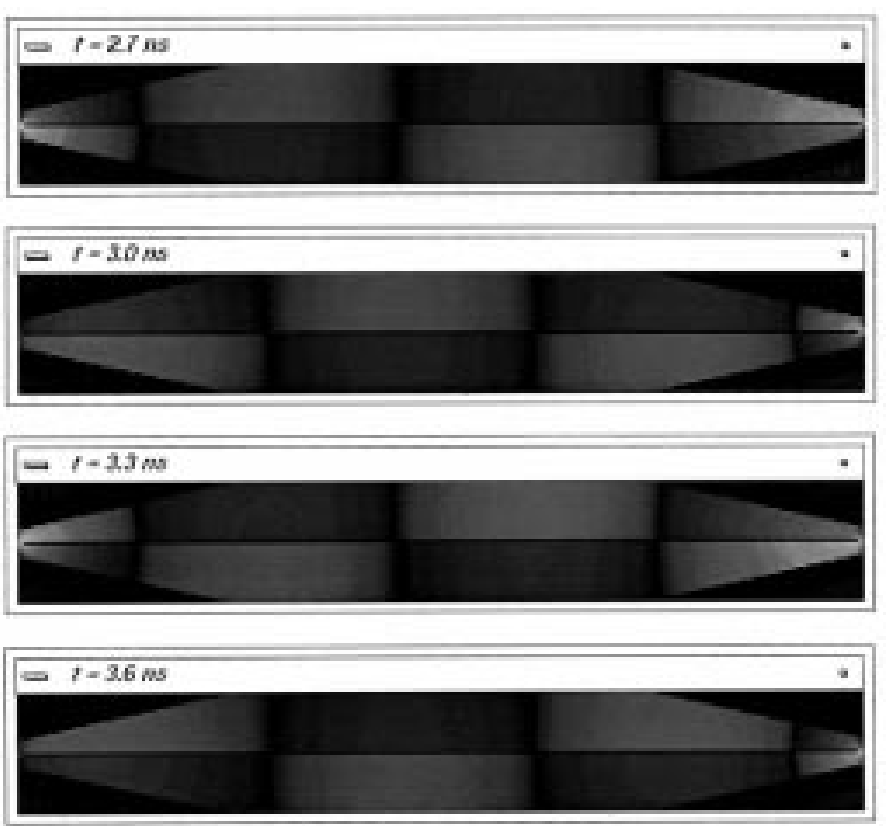

Fig. 2. Gray-scale visualizations of the electric field $E_{z}$ (perpendicular to the septum) within the empty TEM cell at one-quarter-cycle time intervals. These snapshots were taken after the system reached the sinusoidal steady state.

used to solve Maxwell's equations for the TEM cell geometry. After a decade of exponential growth of FDTD publications beginning in 1985, FDTD is considered to be one of the most popular and robust means to implement engineering electromagnetics models [8]. Beginning with the study of microwave absorption within the human eye [9], FDTD has been an increasingly popular tool to study electromagnetic wave interactions with biological tissues for both the impulsive and time-harmonic cases [10], [11]. Such bio-electromagnetics studies have generally been in the context of partial-body or whole-body simulations of humans exposed to field sources ranging from power lines to $\mathrm{RF}$ and microwave antennas to sources of visible light [12], [13].

\section{EMPTY TEM CELL MODEL}

As shown in Fig. 1, the TEM cell consists of a section of rectangular coaxial transmission line tapered at each end to adapt to standard coaxial connectors [1]. There are several important considerations in the TEM cell design. First, the cross section of the test area available for use should be maximized. Second, the upper frequency limit for the desired mode of operation should be as high as possible. Third, the TEM cell impedance should be matched in order to minimize the field standing wave. Fourth, the electromagnetic field pattern within the TEM cell should be as uniform as possible [1].

The detailed dimensions of the TEM cell implemented in the FDTD model, shown in Fig. 1(b), are based on the design used by the bioelectromagnetics group of the University of Washington [14]. The system operates at a frequency of 837 $\mathrm{MHz}$ for simulation of exposure to cellphone emissions. In the FDTD model, the tapered sections of the outer conductor and the septum are resolved to $1 \mathrm{~mm}$ using the staircase approximation. At this grid resolution, the space increment is

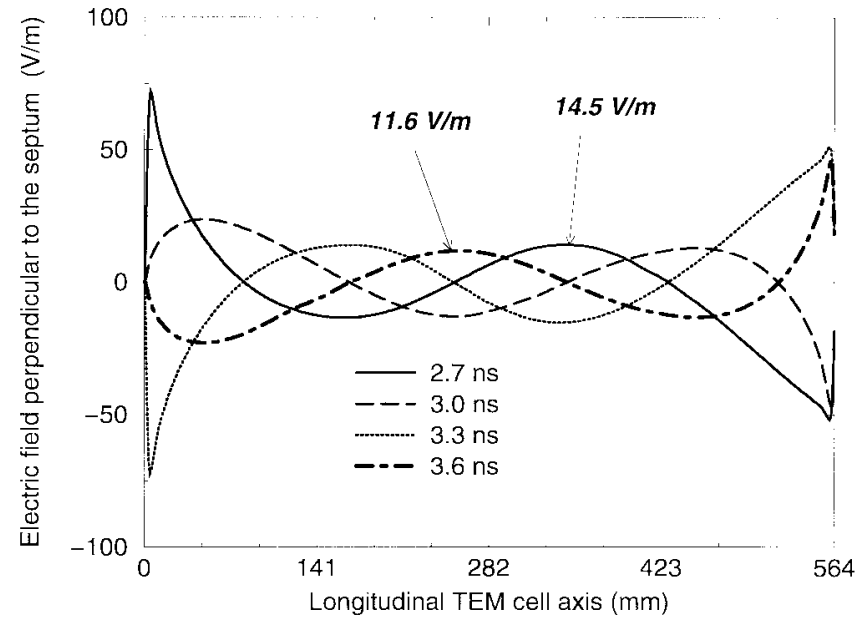

Fig. 3. Line graphs of the electric field $E_{z}$ (perpendicular to the septum) obtained from the gray-scale visualizations of Fig. 2. The curves are one-quarter cycle apart in time and show a field SWR $\approx 1.25$.

TABLE I

Materials Used in the Model and Their Electrical Properties. Averaged Values Were Used for Electric Field Components on the Boundaries of Two or More Materials

\begin{tabular}{|c|c|c|}
\hline Material & $\varepsilon_{\mathrm{r}}$ & $\sigma(\mathrm{S} / \mathrm{m})$ \\
\hline Free space & 1.0 & 0.0 \\
\hline Metal & 1.0 & $3.27 \times 10^{7}$ \\
\hline Polystyrene & 2.55 & 0.0 \\
\hline Cell culture & 72.73 & 1.63 \\
\hline Borosilicate glass & 4.83 & 0.0 \\
\hline $\begin{array}{c}\text { Average: polystyrene } \mathbf{5 0 \%}, \\
\text { air } \mathbf{5 0 \%}\end{array}$ & 1.77 & 0.0 \\
\hline $\begin{array}{c}\text { Average: polystyrene } 50 \% \\
\text { cell culture } 50 \%\end{array}$ & 37.64 & 0.82 \\
\hline $\begin{array}{l}\text { Average: air } 50 \%, \\
\text { cell culture } 20 \%, \\
\text { borosilicate glass } 30 \%\end{array}$ & 16.49 & 0.33 \\
\hline $\begin{array}{r}\text { Average: air } \mathbf{5 0 \%}, \\
\text { polystyrene } 25 \% \\
\text { cell culture } 17.5 \% \\
\text { borosilicate glass } 7.5 \%\end{array}$ & 14.23 & 0.29 \\
\hline
\end{tabular}

$\lambda_{0} / 358$ (where $\lambda_{0}$ is the free-space wavelength). This resolution is so fine that artifacts due to the staircase approximation are negligible, and there is no need for usage of conformal gridding.

A sinusoidal excitation of this model at $837 \mathrm{MHz}$ is provided by programming a $1-\mathrm{V}, 50-\Omega$ resistive voltage source [15] in free space between the septum and the outer tapered walls at the narrowest point of one of the transition sections. The other tapered section is assumed to be terminated with a matched $50-\Omega$ load using the lumped-circuit-element model [15].

The effectiveness of the empty TEM cell design was verified by running the 3-D FDTD model. The empty TEM cell should 


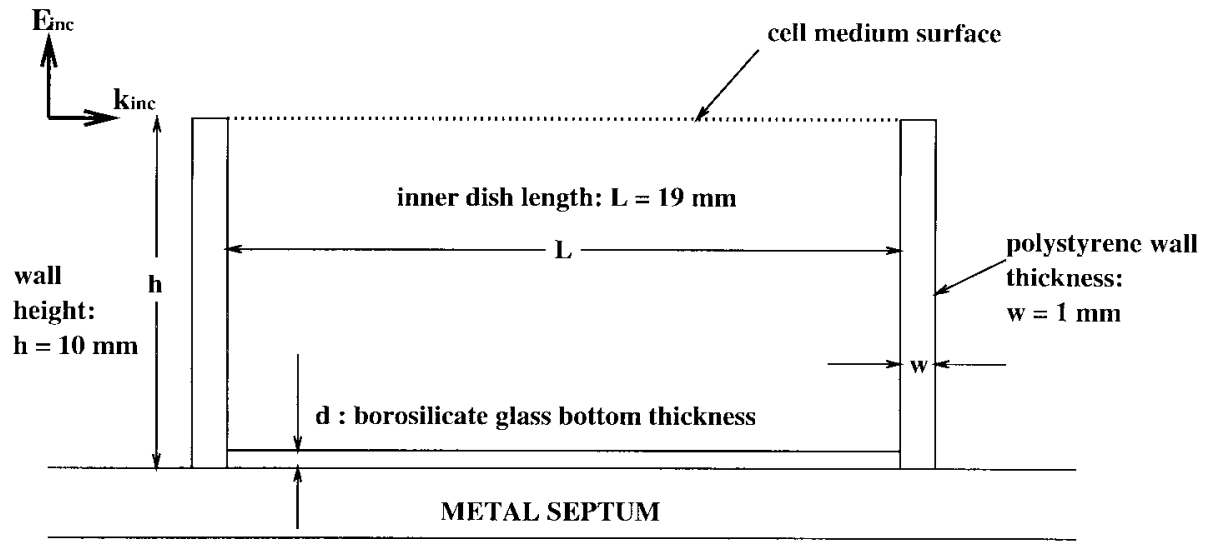

(a)

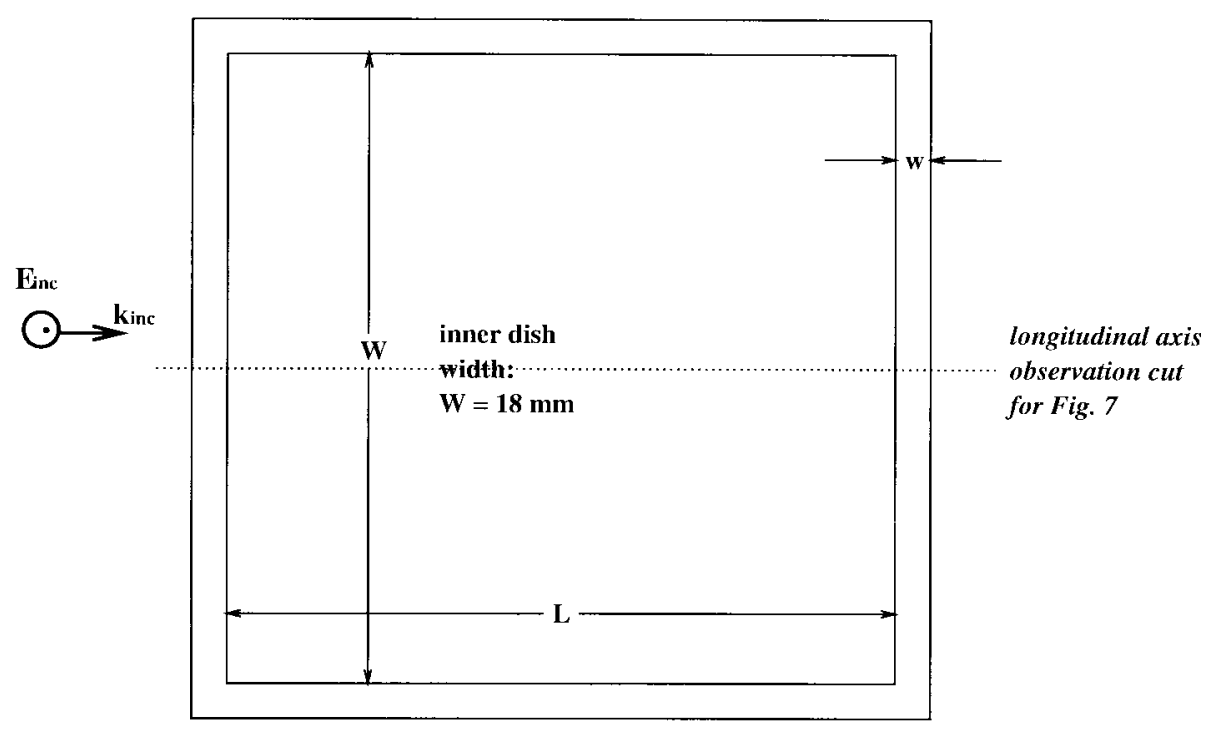

(b)

Fig. 4. One-dish geometry considered for the study of the effect of the glass-bottom thickness $d$ on the SAR distribution. (a) Longitudinal cross section. (b) Plan view. The FDTD model was run for three values of d: $0 \mathrm{~mm}$ (no glass bottom); $0.3 \mathrm{~mm}$ (correct glass bottom thickness); and $1 \mathrm{~mm}$ (one grid cell).

exhibit low standing wave ratio and good field uniformity in each transverse plane. Fig. 2 shows gray-scale visualizations of the $E_{z}$ field component (perpendicular to the septum) within the central longitudinal plane of the TEM cell after the system attains the sinusoidal steady state. The snapshots are taken at intervals of one-quarter cycle at $837 \mathrm{MHz}$. We see the expected motion of the field pattern from source to load (left to right) and the expected odd symmetry of the field direction with respect to $z$ above and below the septum.

Fig. 3 allows determination of the standing wave ratio (SWR) of $E_{z}$ along the longitudinal axis of the empty TEM cell. The four curves here are snapshots of the $E_{z}$ distribution taken one-quarter cycle apart in time at $837 \mathrm{MHz}$. In fact, these curves are obtained from the 2-D plots shown in Fig. 2 by plotting field values along the longitudinal axis and $4 \mathrm{~mm}$ above the septum. The longitudinal coordinate origin is the voltage source point. Fig. 3 indicates that there exists a low $\mathrm{SWR} \approx 1.25$, which confirms the design of the empty TEM cell from the standpoint of impedance matching and field uniformity along the septum.

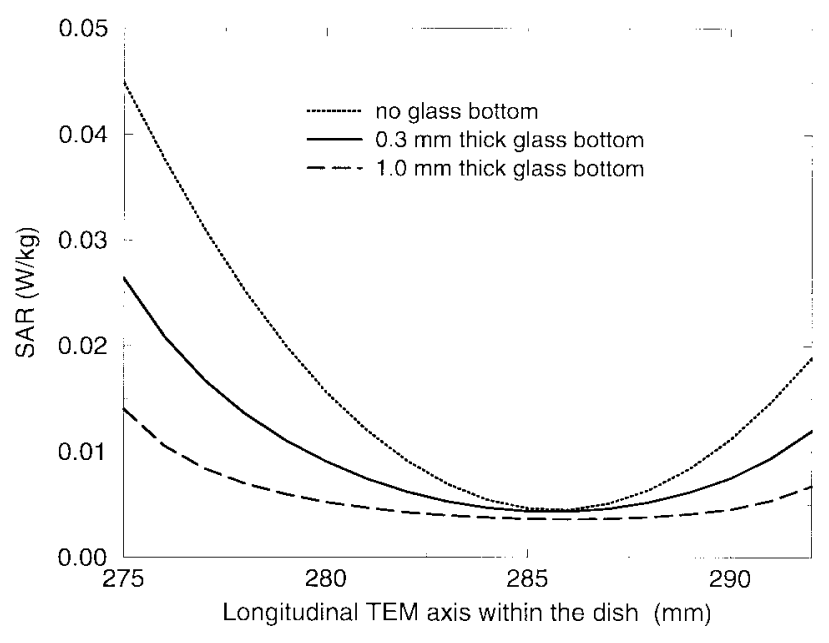

Fig. 5. Line graphs of the FDTD-computed SAR within the cell culture in a single culture dish placed on the septum in the center of the TEM cell. These data are obtained along the central longitudinal cut defined in Fig. 4 (b), $3 \mathrm{~mm}$ above the septum. 


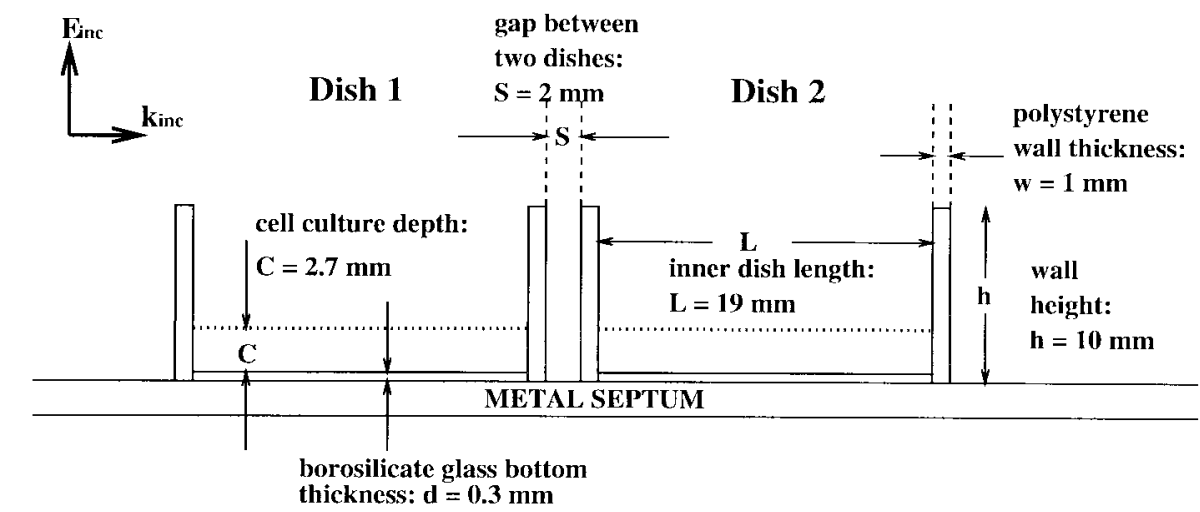

(a)

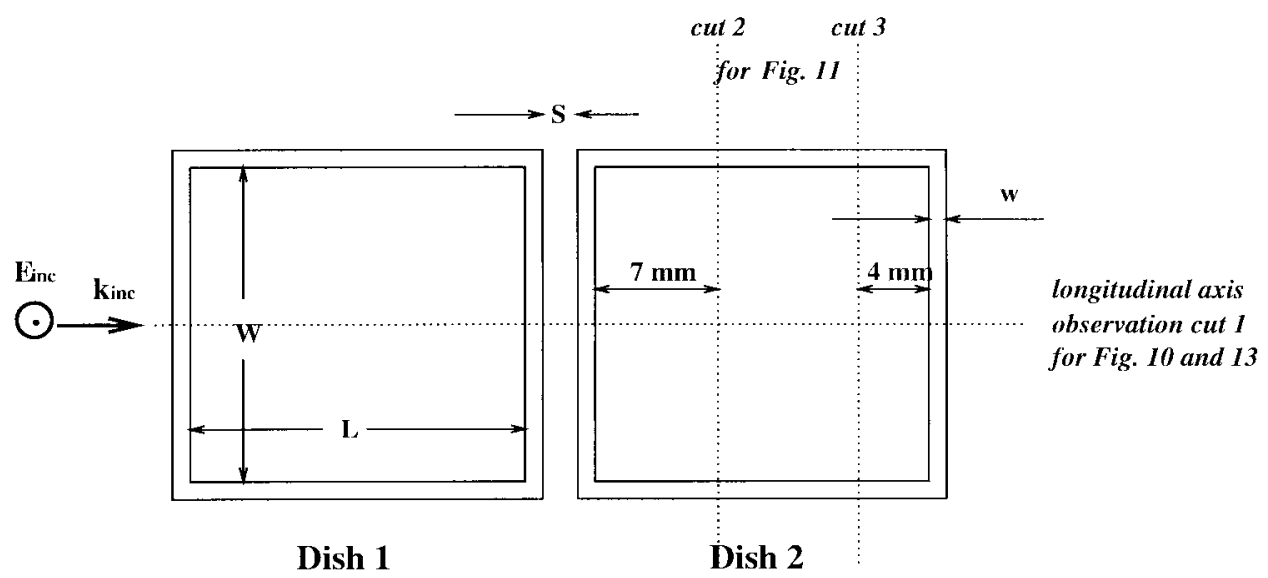

(b)

Fig. 6. The geometry used in the two-dish FDTD model. The two dishes are centered on the septum within the TEM cell along its longitudinal axis. (a) Longitudinal cross section. (b) Plan view.

\section{Definition of Media Parameters AND SPECIFIC ABSORPTION RATE}

The FDTD model of the TEM cell loaded with culture dishes requires specification of the electrical properties of all materials involved in the geometry [14]. Table I provides a list of each material and its assumed relative permittivity $\epsilon_{r}$ and conductivity $\sigma$. Because some electric field components lie on the boundaries of two or more dissimilar media, these are assigned weighted-average electrical properties.

The envelopes of the electric field components of interest located within distinct cross section planes of the grid are obtained using discrete Fourier transforms conducted simultaneously with the FDTD time stepping. Once the envelopes of the desired field components at these planes are calculated, the SAR can be computed. We first define the heating potential $Q$

$$
Q=0.5 \sigma|\boldsymbol{E}|^{2}=0.5 \sigma\left(E_{x}^{2}+E_{y}^{2}+E_{z}^{2}\right) \quad \mathrm{W} / \mathrm{m}^{3}
$$

Then, we define the SAR as

$$
\mathrm{SAR}=\frac{Q}{\varrho_{\mathrm{H}_{2} \mathrm{O}}} \quad \mathrm{W} / \mathrm{kg}
$$

where $\varrho_{\mathrm{H}_{2} \mathrm{O}}=1000 \mathrm{~kg} / \mathrm{m}^{3}$ is the density of water.

\section{Modeling Results for One Culture Dish}

The measured thickness of the glass bottom of a culture dish is $0.3 \mathrm{~mm}$, much less than the 1-mm unit-cell size of the lattice used for the FDTD model. It was desired to determine what effect, if any, the precise thickness of the glass bottom has upon the SAR of the culture medium. A contour-path method to model a subcell material sheet perpendicular to a major axis of the FDTD grid in three dimensions was applied for this purpose [16]. FDTD models were run for three glass-bottom thicknesses: zero (no glass bottom); $0.3 \mathrm{~mm}$ (the correct thickness); and $1 \mathrm{~mm}$ (one grid cell). In all cases, the dish was assumed to be fully filled with culture medium.

The culture dish geometry modeled for this investigation is shown in Fig. 4 in two views, a longitudinal cross section and a plan view. The dish was assumed to be centered on the septum of the TEM cell along its longitudinal axis. The transverse coordinate origin is the side wall of the TEM cell. Fig. 5 graphs SAR values along the central longitudinal axis [observation cut of Fig. 4(b)] $3 \mathrm{~mm}$ above the septum, for all thicknesses of the glass bottom under investigation. These results indicate a substantial nonuniformity of the SAR in the longitudinal direction. For any particular glass-bottom thickness, the highest SAR values are located next to the dish walls, and the lowest SAR values are located near the center of the dish. Further, the assumed glass-bottom thickness is seen to 


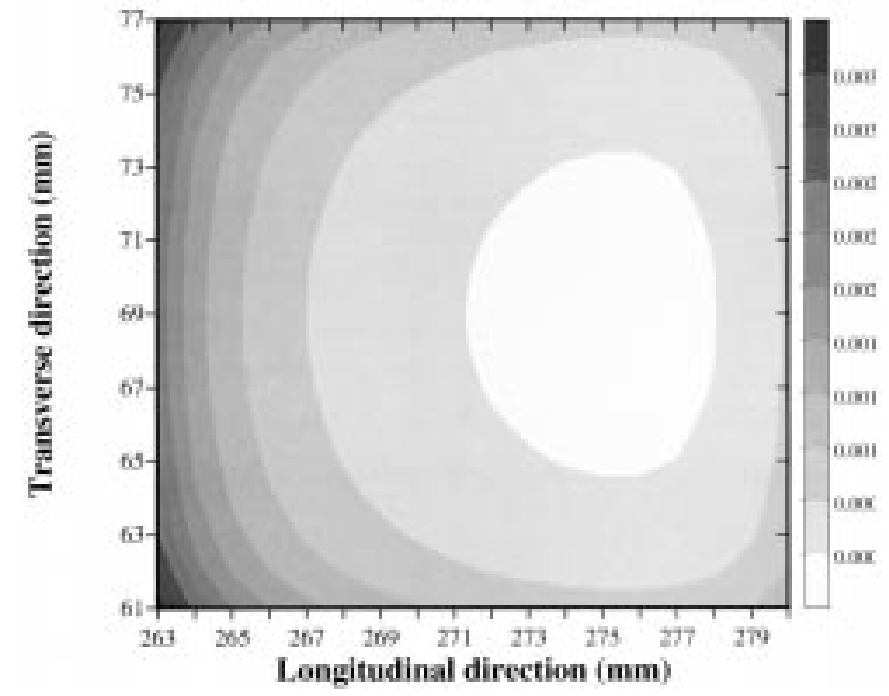

(a)

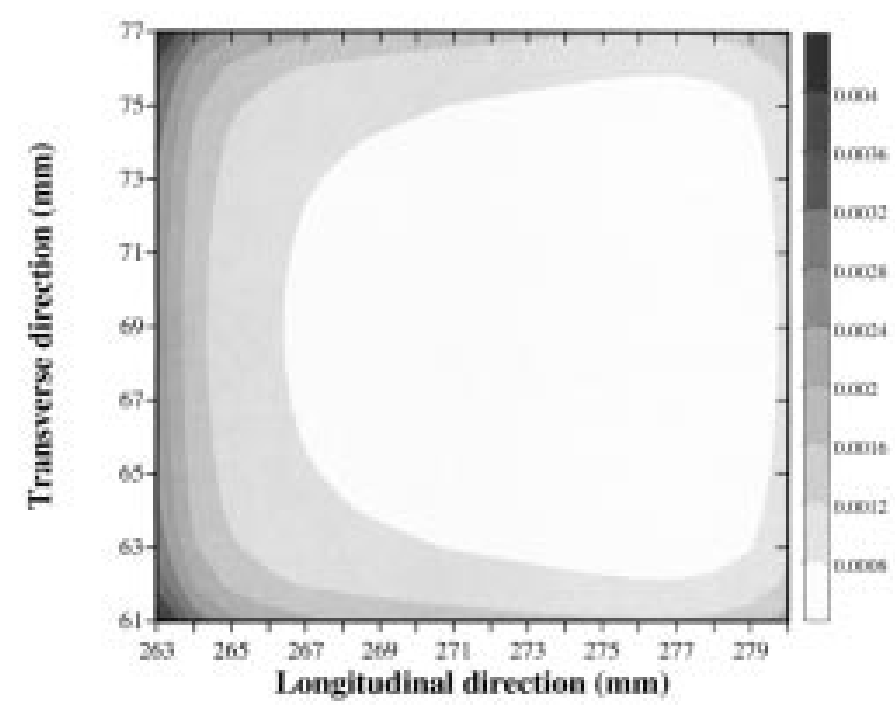

(c)

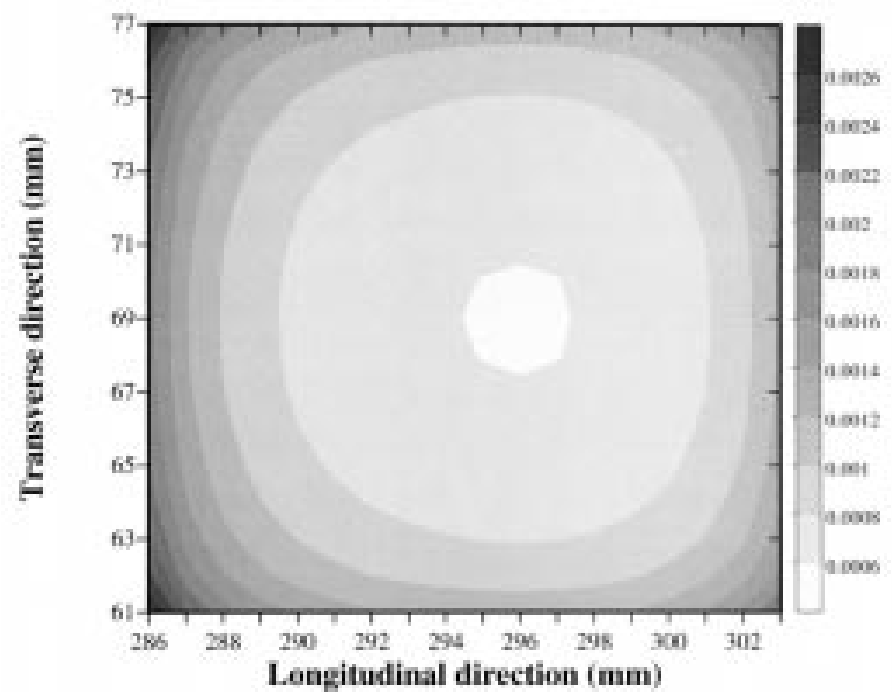

(b)

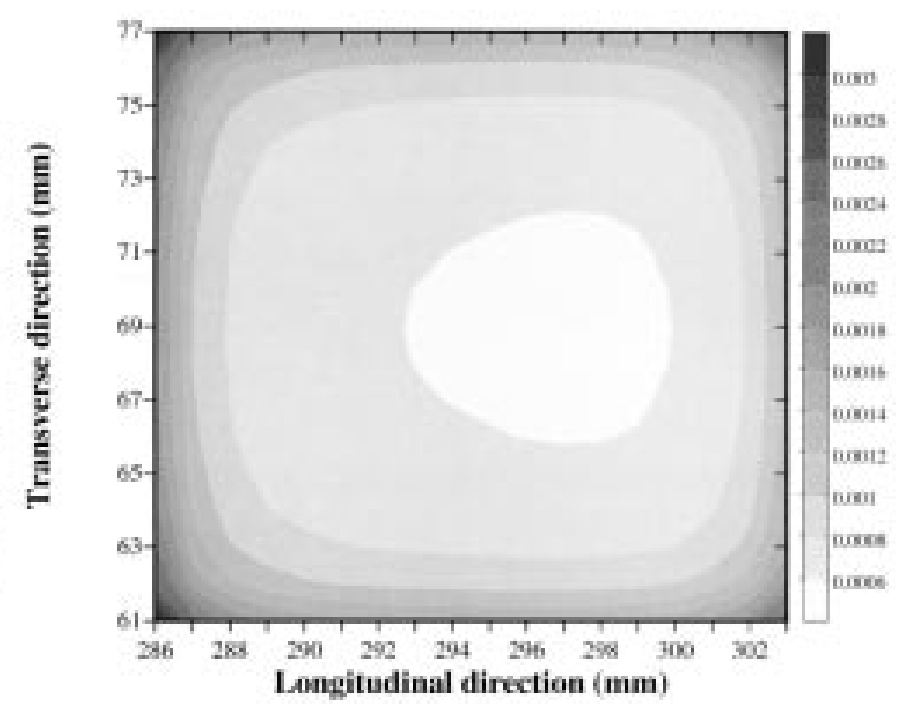

(d)

Fig. 7. Gray-scale visualization of the FDTD-computed SAR (W/kg) within the cell culture in the two-dish model of Fig. 6. The view is that of the Fig. 6(b). (a) Dish 1, $0.7 \mathrm{~mm}$ above glass bottom. (b) Dish 2, $0.7 \mathrm{~mm}$ above glass bottom. (c) Dish 1, $1.7 \mathrm{~mm}$ above glass bottom. (d) Dish 2, $1.7 \mathrm{~mm}$ above glass bottom. For all four visualizations, the incident field is propagating from left to right.

markedly affect the SAR. These results justify the usage of the precise 0.3-mm sub-cell glass bottom model in all subsequent studies reported in this paper.

\section{Modeling Results For Two Culture Dishes}

A standard biological specimen culture container used in experiments with the TEM cell exposure system was obtained for examination [14]. The standard container consists of two culture dishes of the type shown in Fig. 4 located side by side and connected by a thin dielectric structure. The connecting material between the dishes was omitted in the FDTD model. Fig. 6 depicts the two-dish geometry as incorporated in the FDTD model. The two-dish geometry was assumed to be centered on the septum of the TEM cell along its longitudinal axis. An additional step toward modeling the actual experiment as accurately as possible was to assume that the culture dishes were only partially filled with the cell medium to a depth of $2.7 \mathrm{~mm}$. The liquid surface was assumed to be planar.

Fig. 7 is the gray-scale visualization of the 2-D SAR distribution within the culture medium along two observation planes parallel to the septum, 0.7 and $1.7 \mathrm{~mm}$ above the bottom of the culture dishes. Fig. 8(a) shows the corresponding SAR along the central longitudinal axis [Cut 1 of Fig. 6(b)], while Fig. 8(b) shows the SAR along transverse Cuts 2 and 3. Pronounced nonuniformities of the SAR are detected. Large SAR peaks are located at the corners of the dishes and near the front and back walls. Ratios of maximum to minimum SAR for the data shown in Fig. 7 are as high as 8.2:1.

\section{Modeling Results for the Meniscus EFFECT}

Molecular interactions of the liquid culture medium and the polystyrene walls, combined with intermolecular interactions 


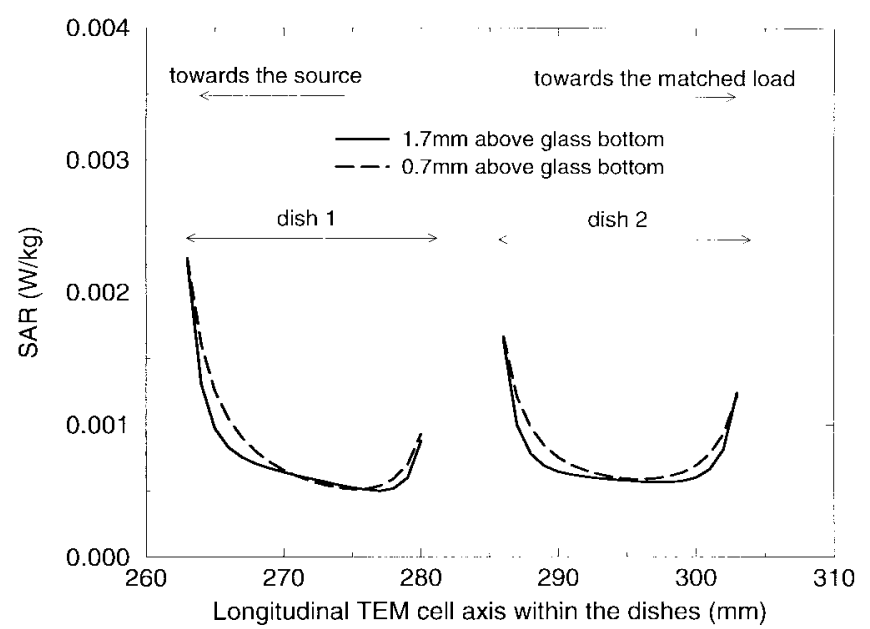

(a)

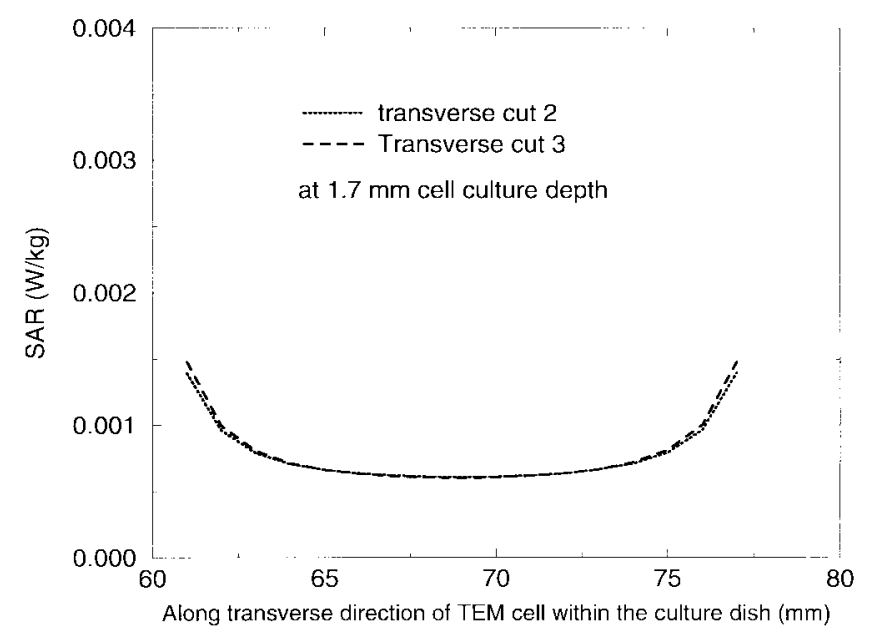

(b)

Fig. 8. Line-graphs of the FDTD-computed SAR within the cell culture in the two-dish model of Fig. 6 along (a) Cut 1 and (b) Cuts 2 and 3 at 1.7 $\mathrm{mm}$ above the glass bottom. These data are obtained from the 2-D SAR visualizations of Fig. 7.

in the liquid itself, cause a curved surface (meniscus) of the culture medium. Here, we investigate the impact of the meniscus on the SAR distribution within the two-culture-dish exposure geometry of Section VI.

Measured data for the depth (in $\mathrm{mm}$ ) of the culture medium were provided for one-half of a culture dish [14]. Even symmetry of the depth distribution was assumed for the other half. The total volume of the culture medium here is approximately that of the no-meniscus medium considered in Section VI. The depths are measured with a precision of 0.1 $\mathrm{mm}$, which is much finer than the FDTD grid resolution (1 $\mathrm{mm})$. For simplicity, the culture-medium depth at each point is approximated as an integer number of FDTD grid cells. It is believed that this simple model adequately addresses the physical impact of the curved liquid surface. The approximated culture-depth distribution within each of the two dishes is shown in Fig. 9.

The SAR distribution within the culture medium for the two-dish model which includes the meniscus effect is shown in Fig. 10. These data are taken along the central longitudinal

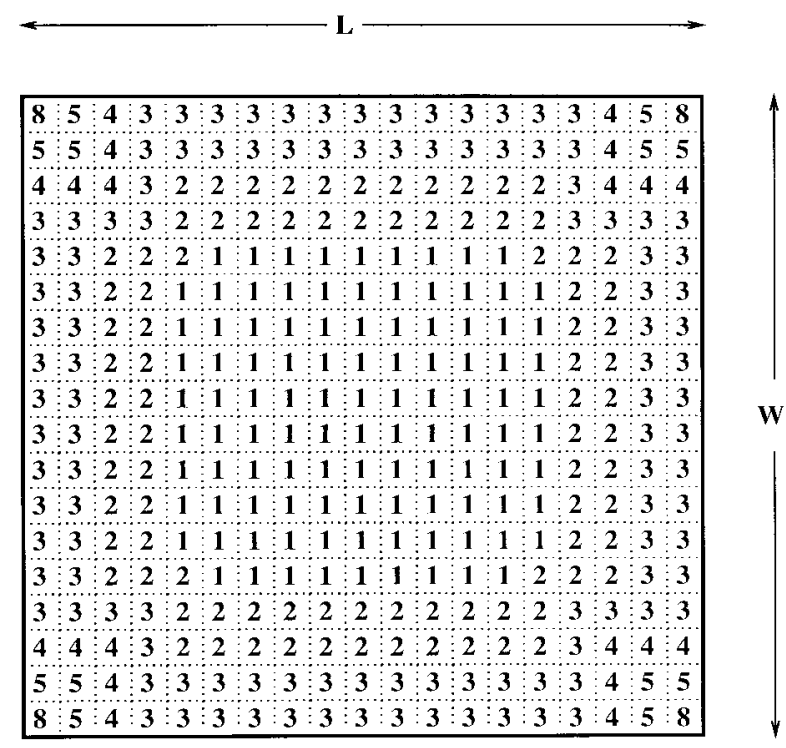

Fig. 9. The geometry used in the two-dish FDTD model incorporating the meniscus. The figure shows approximation of the cell culture depth as an integer number of FDTD grid cells.

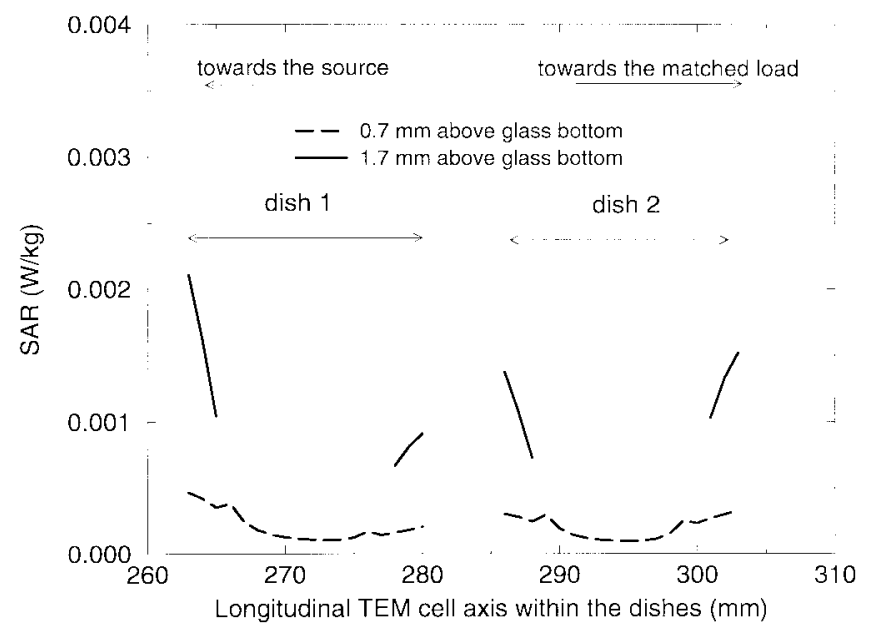

Fig. 10. Line-graphs of the FDTD-computed SAR within the cell culture in the two-dish model of Fig. 6 along Cut 1 including the meniscus of Fig. 9.

axis [Cut 1 of Fig. 6(b)] of the two-dish geometry at 0.7 and $1.7 \mathrm{~mm}$ above the glass bottom. It is seen that the SAR nonuniformity is even more pronounced than in the previous no-meniscus case of Fig. 8(a). The nonuniformity problem is worsened by the fact that excess liquid accumulates adjacent to the dish walls in the high-SAR zone.

\section{Modeling Results for THE Vertical TEM Cell}

The studies described in the previous sections demonstrated a substantial nonuniformity of the SAR distribution within the culture medium. This section explores possible means to mitigate the SAR nonuniformity by reorienting the culture dishes. Here, the TEM cell is oriented vertically, and the culture dishes are placed on a dielectric holder perpendicular to the septum. This allows the incident wave to propagate in a direction perpendicular to the surface of the liquid culture 


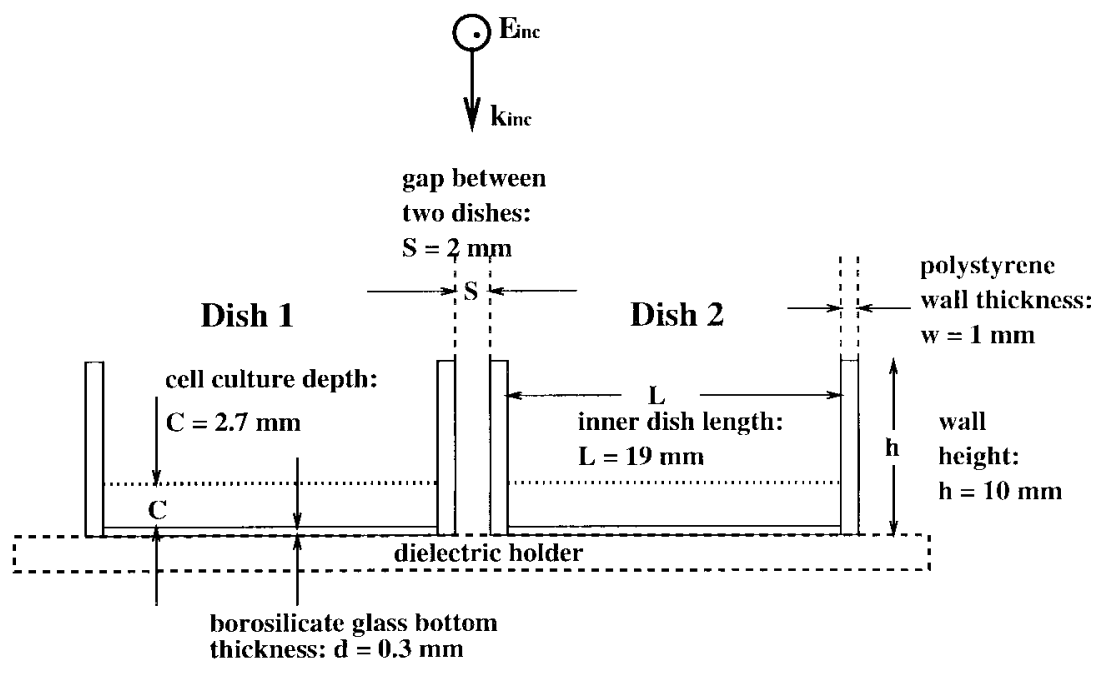

(a)

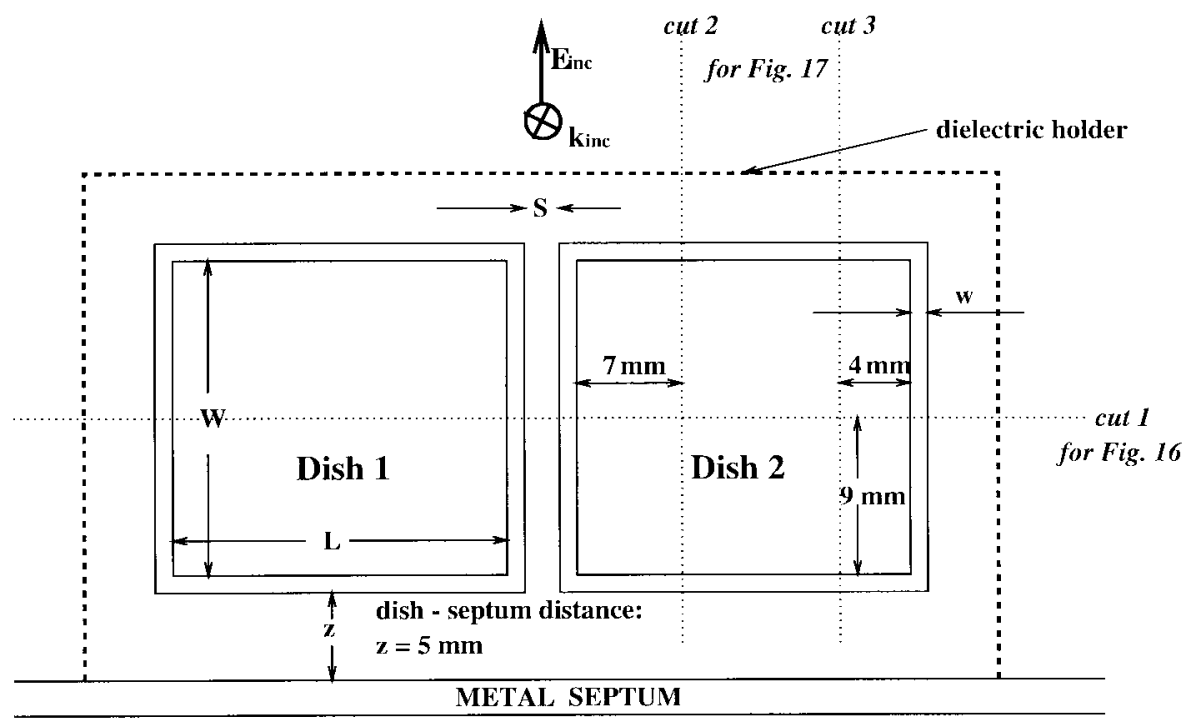

(b)

Fig. 11. The geometry used in the two-dish FDTD model in the vertical TEM cell. The surface of the culture medium is now perpendicular to the septum. (a) Cross section parallel to the septum. (b) Plan view.

medium. No meniscus is assumed. The reoriented geometry is shown in Fig. 11.

Fig. 12 provides gray-scale visualizations of the FDTDcomputed SAR within the culture medium of one of the containers in the two-dish model of Fig. 11. The data show SAR at two planes: 0.7 and $1.7 \mathrm{~mm}$ above the glass bottom. The SAR distribution for the second dish is a mirror image of the SAR shown for the first one due to even symmetry of the geometry with respect to the propagating wave. The distance along the transverse direction parallel to the septum is measured from the side of the TEM cell. The septum serves as the reference point for the measurements along the transverse direction perpendicular to it. We observe a very different pattern from that of Fig. 7. To enable a line-graph comparison between the two cases, data taken along Cut 1 defined in Fig. 11(b) are graphed in Fig. 13(a). It is seen that the nonuniformity in the direction of Cut 1 is substantially reduced relative to that of Fig. 10. However, Fig. 12 suggests that, although suppressed along Cut 1, the nonuniformity of the SAR distribution increases along Cuts 2 and 3 of Fig. 11(b). This is shown in Fig. 13(b).

Overall, the vertical TEM cell provides some improvement in the ratio of maximum-to-minimum SAR, which does not exceed $6.4: 1$ for this case. This ratio is still quite high and must be accounted for in the biological response-versus-dose studies.

\section{CONCLUSION}

This paper reported FDTD modeling of the exposure of liquid biological cultures to an impinging $837-\mathrm{MHz}$ electromagnetic wave within the TEM cell. As an initial step, the FDTD model was run for the empty TEM cell and verified the low SWR and uniform fields of the basic exposure system. A high-resolution (uniform 1-mm) FDTD grid augmented by a subcell model of the culture-dish glass-bottom plate was 


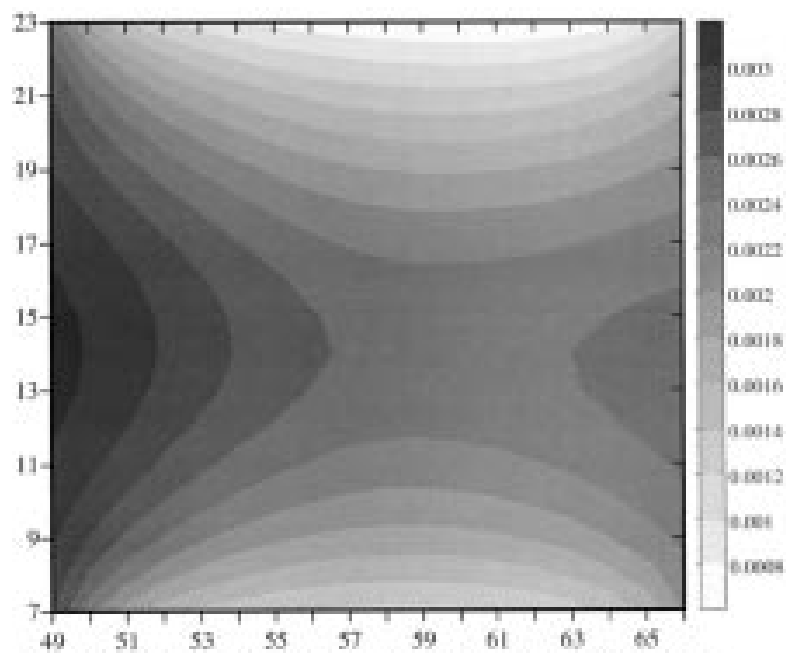

Transverse direction parallel to the septam ( $\mathrm{mm}$ )

(a)

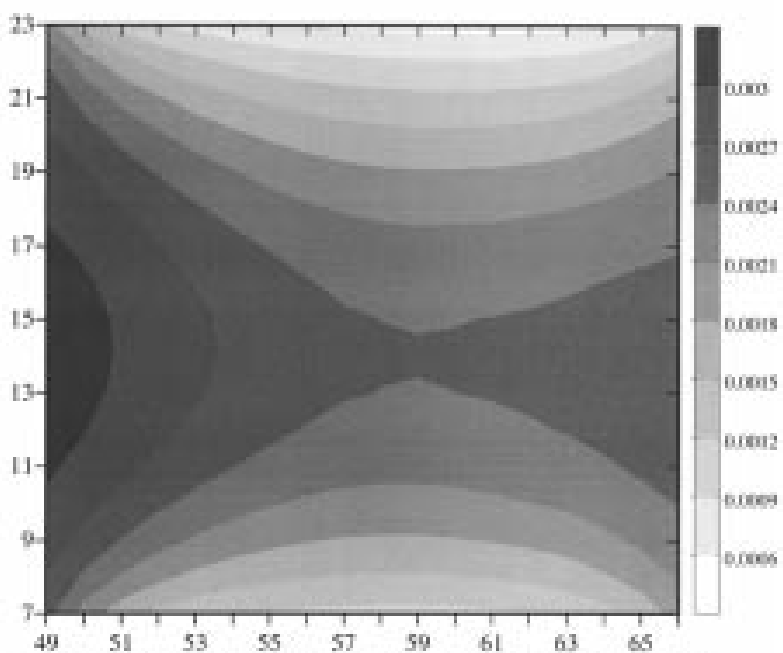

Transverse direction parallel to the septum (mm)

(b)

Fig. 12. Gray-scale visualization of the FDTD-computed SAR (W/kg) in the two-dish model of Fig. 11. (a) Dish 1, $0.7 \mathrm{~mm}$ above glass bottom. (b) Dish 1, $1.7 \mathrm{~mm}$ above glass bottom. The SAR distribution in Dish 2 shows complete mirror-symmetry to that of the Dish 1 . For all visualizations, the incident field is propagating perpendicular to the plane of the page.

sufficient to render several geometries and orientations of the culture dishes containing liquid medium. For each case studied, the SAR distribution within the culture liquid was computed as the indication of electromagnetic energy dosage. All cases studied exhibit a high ratio of maximum-to-minimum SAR, which must be taken into account in related biological dosimetric studies. Dose-response experiments using the TEM cell should account for the possibility of strong SAR peaking in the culture medium to provide useful data in setting exposure standards for wireless communications. This work also appears to open possibilities for anatomical models of

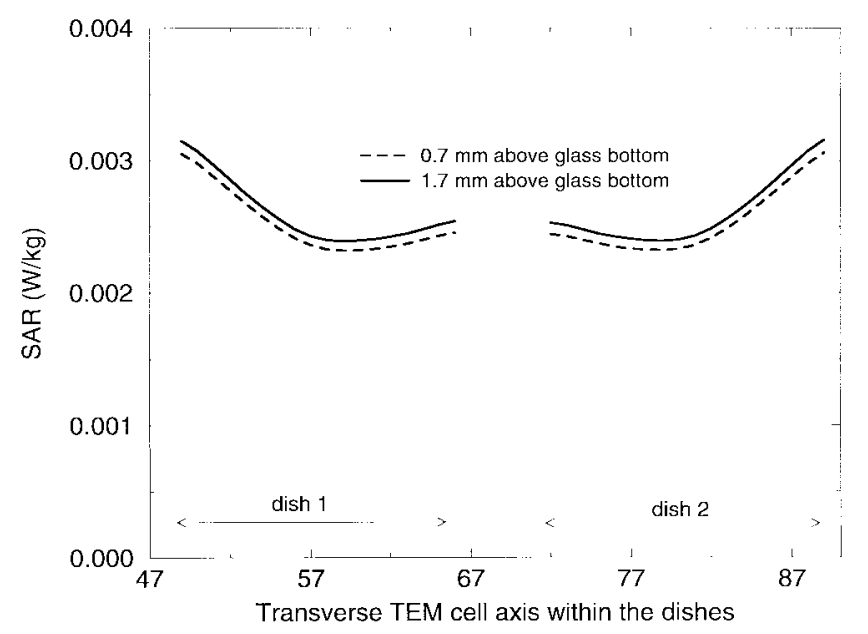

(a)

0.004

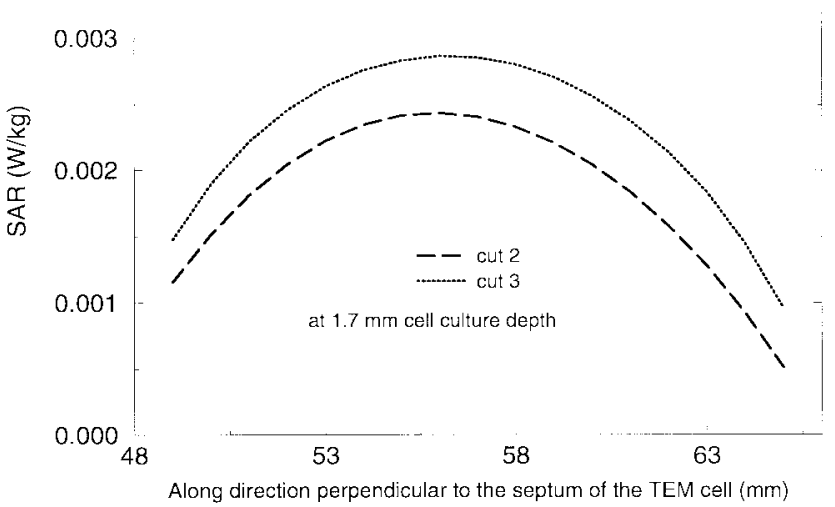

(b)

Fig. 13. Line graphs of the FDTD-computed SAR in the two-dish model of Fig. 11 along (a) Cut 1 and (b) Cuts 2 and 3 at $1.7 \mathrm{~mm}$ above the glass bottom. These data are obtained from the 2-D SAR visualizations of Fig. 12.

small animals within TEM cells, the logical next level of FDTD investigations relevant to current laboratory work in the wireless exposure health area.

\section{ACKNOWLEDGMENT}

The authors acknowledge the assistance of Dr. A. W. Guy of the University of Washington in this work.

\section{REFERENCES}

[1] M. L. Crawford, "Generation of standard EM fields using TEM transmission cells," IEEE Trans. Electromag. Compat., vol. 16, no. 4, pp. 189-195, Nov. 1974.

[2] J. Van Hese, L. Maartens, D. De Zutter, C. De Wagter, L. O. G. Malmgren, B. R. R. Persson, and L. G. Salford, "Simulation of the effect of inhomogeneities in TEM transmission cells using the FDTD-method," IEEE Trans. Electromag. Compat., vol. 34, no. 3, pp. 292-298, 1992.

[3] L. Martens, J. Van Hese, D. De Zutter, C. De Wagter, L. Malmgren, B. R. R. Persson, and L. G. Salford, "Electromagnetic field calculations used for exposure experiments on small animals in TEM-cells," Bioelectrochem. Bioenergetics, no. 30, pp. 73-81, 1993.

[4] K. S. Yee, "Numerical solution of initial boundary value problems involving Maxwell's equation in isotropic media," IEEE Trans. Antennas Propagat., vol. 14, pp. 302-307, 1966. 
[5] K. S. Kunz and R. J. Luebbers, The Finite Difference Time Domain Method for Electromagnetics. Boca Raton, FL: CRC Press, 1993.

[6] A. Taflove, Computational Electrodynamics: The Finite-Difference Time-Domain Method. Norwood, MA: Artech House, 1995.

[7] Cai-XD and Costache-GI, "An engineering design approach of a tripleTEM cell using finite-element method," IEEE Trans. Electromag. Compat., vol. 16, no. 4, pp. 189-195, Nov. 1974.

[8] K. L. Shlager and J. B. Schneider, "A selective survey of the FiniteDifference Time-Domain literature," IEEE Antennas Propagat. Mag., vol. 37, no. 4, pp. 39-57, 1995

[9] A. Taflove and M. E. Brodwin, "Computation of the electromagnetic fields and induced temperatures within a model of the microwaveirradiated human eye," IEEE Trans. Microwave Theory Tech., vol. 23, pp. 888-896, 1975.

[10] D. M. Sullivan, O. P. Gandhi, and A. Taflove, "Use of the finitedifference time-domain method in calculating EM absorption in man models," IEEE Trans. Biomed. Eng., vol. 35, pp. 179-186, 1988.

[11] P. J. Dimbylow and O. P. Gandhi, "Finite-difference time-domain calculations of SAR in realistic heterogeneous model of the head for plane-wave exposure from $600 \mathrm{MHz}$ to $3 \mathrm{GHz}$," Phys. Med. Biolol., vol. 36, pp. 1075-1089, 1991.

[12] M. J. Piket-May, A. Taflove, W. C. Lin, D. S. Katz, V. Sathiaseelan, and B. B. Mittal, "Initial results for automated computational modeling of patient-specific electromagnetic hyperthermia," IEEE Trans. Biomed. Eng., vol. 39, pp. 226-237, 1992.

[13] M. J. Piket-May, A. Taflove, and J. B. Troy, "Electrodynamics of visible light interactions with the vertebrate retinal rod," Opt. Lett., vol. 18, pp. 568-570, 1993

[14] A. W. Guy, private communication, Univ. Washington, Seattle, 1995.

[15] M. J. Piket-May, A. Taflove, and J. Baron, "FD-TD modeling of digital signal propagation in 3-D circuits with passive and active loads," IEEE Trans. Microwave Theory Tech., vol. 42, pp. 1514-1523, 1994.

[16] J. G. Maloney and G. S. Smith, "The efficient modeling of thin material sheets in the finite-difference time-domain method," IEEE Trans. Antennas Propagat., vol. 40, pp. 323-330, 1992.

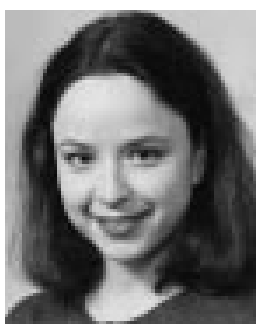

Milica Popović (S'94) was born in Radford, VA, on March 9, 1969. She received the B.S. degree in electrical engineering from University of Colorado, Boulder, in 1994. In 1997, she received the M.S. degree in electrical engineering from Northwestern University, IL, where she is currently pursuing the $\mathrm{Ph} . \mathrm{D}$. degree in the same area.

She is currently interested in researching computational electromagnetics and applications in biology and medicine.

Ms. Popović won the Teaching Assistant of the Year Award from the Electrical and Computer Engineering Department at Northwestern University in 1997. She is a member of Eta Kappa Nu.

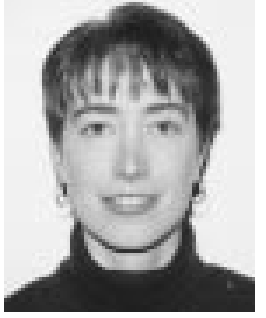

Susan C. Hagness (S'91-M'98) was born in Terre Haute, IN, on December 16, 1970. She received the B.S. degree with highest distinction and the Ph.D degree in electrical engineering from Northwestern University, Evanston, IL, in 1993 and 1998, respectively.

She joined the Department of Electrical and Computer Engineering at the University of WisconsinMadison, Madison, as an Assistant Professor in August 1998. Her current research interests are in FDTD algorithm development and analysis for biomedical and optical applications, including the detection and imaging of early-stage breast cancer using nonionizing electromagnetic waves and micron-scale integrated photonic devices and circuits for applications in optical communications and computing. She has published over 30 refereed journal and conference papers.

In 1992, Dr. Hagness was the recipient of the Eta Kappa Nu Norman R. Carson Award as the Outstanding Junior Electrical Engineering Student in the United States. In 1993 she received a National Science Foundation Graduate Fellowship and was named a Tau Beta Pi Spencer Fellow. She was awarded a Northwestern University Cabell Dissertation-Year Fellowship in 1997 and the Department of Electrical and Computer Engineering Best Dissertation Award in 1998. She received the Student Paper Competition Third Prize Award at the 1998 IEEE AP-S International Symposium and URSI Radio Science Meeting. She is a member of Tau Beta Pi, Etta Kappa Nu, the Optical Society of America, and the American Society for Electrical Engineering Education.

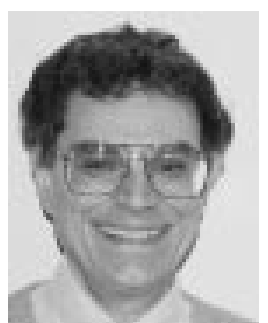

Allen Taflove (F'90) was born in Chicago, IL, on June 14, 1949. He received the B.S., M.S., and $\mathrm{Ph} . \mathrm{D}$. degrees in electrical engineering from Northwestern University, Evanston, IL in 1971, 1972, and 1975, respectively.

After nine years as a Research Engineer at ITT Research Institute, Chicago, IL, he returned to Northwestern University in 1984, and since 1988 he has been a Professor in the Department of Electrical and Computer Engineering. Since 1990, he has given many invited talks and lectures in the United States and abroad on FDTD and horizons in computational electromagnetics. $\mathrm{He}$ is the author of Computational Electrodynamics: The Finite-Difference Time-Domain Method (Norwood, MA: Artech House, 1995) and the Editor of Advances in Computational Electrodynamics: The Finite-Difference TimeDomain Method (Norwood, MA: Artech House, 1998). His current research interests include FDTD simulations of VLSI-scale optoelectronic devices, pulsed confocal microwave technology for detection of early stage breast cancers, and diffraction of cellular radio waves by the corners and rooftops of realistic buildings.

Dr. Taflove was a Distinguished National Lecturer for the IEEE Antennas and Propagation Society in 1990-1991, and in 1992 was Chairman of the Technical Program of the IEEE Antennas and Propagation Society International Symposium in Chicago, IL. He is a member of Eta Kappa Nu, Tau Beta Pi, Sigma Xi, International Union of Radio Science (Commissions B, D, and K), the Electromagnetics Academy, and the Optical Society of America. His biographical listings include Who's Who in Engineering, Who's Who in America, Who's Who in Science and Engineering, and Who's Who in American Education. 